C-A/AP/\#416 Jan. 2011

\title{
Single Pass Electron Beam Cooling of Gold Ions Between EBIS LINAC and Booster Is Theoretically Possible!
}

\author{
A. Hershcovitch
}

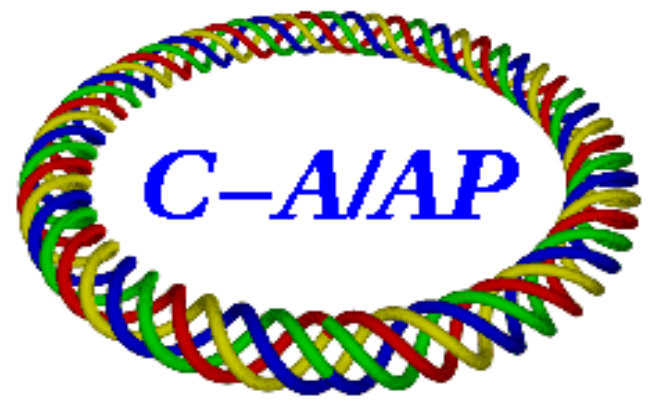

Collider-Accelerator Department Brookhaven National Laboratory

Upton, NY 11973

Notice: This document has been authorized by enployees of Brookhaven Science Associates, LLC under Contract No. DE-AC02-98CH10886 with the U.S. Department of Energy. The United States Government retains a

non-exclusive, paid-up, irrevocable, world-wide license to publish or reproduce the published form of this document, or allow others to do so, for United States Government purposes. 


\title{
Single Pass Electron Beam Cooling of Gold Ions Between EBIS LINAC and Booster Is Theoretically Possible!
}

\author{
Ady Hershcovitch
}

Electron beam cooling is examined as an option to reduce momentum of gold ions exiting the EBIS LINAC before injection into the booster. Electron beam parameters are based on experimental data (obtained at BNL) of electron beams extracted from a plasma cathode. Many issues, regarding a low energy high current electron beam that is needed for electron beam cooling to reduce momentum of gold ions exiting the EBIS LINAC before injection into the booster, were examined. Computations and some experimental data indicate that none of these issues is a show stopper. Preliminary calculations indicate that single pass cooling is feasible; momentum spread can be reduced by more than an order of magnitude in about one meter. Hence, this option cooling deserves further more serious considerations.

\section{Introduction}

After a number of iterations, ${ }^{1-3}$ a possible scheme for reducing momentum of gold ions exiting the EBIS LINAC before injection into the booster via single pass electron beam cooling was developed. In this scenario, gold ion cooling can be achieved by a copropagating $1 \mathrm{KeV}$ electron beam, which is co-injected with a $2 \mathrm{MeV}$ proton beam, in a 2.4 Tesla solenoid. Expected ion beam parameters ${ }^{4}$, based on design, at the exit of the EBIS-RFQ-LINAC system are: energy $2 \mathrm{MeV} / \mathrm{u}$, momentum spread $\Delta p_{\|} / p=10^{-3}$, and $\Delta p_{\perp} / p=5 \times 10^{-3}$, beam diameter $1 \mathrm{~cm}$, and gold ion charge state $\mathrm{Au}^{+32}$, with ion density ${ }^{2}$ at the LINAC exit $\mathrm{n}_{\mathrm{i}}=8 \times 10^{7} \mathrm{~cm}^{-3}$. For electrons to match ion velocity, their energy $\mathrm{U}$ must be about $1 \mathrm{KeV}$. At these energies, ion and electron velocities are about $2 \times 10^{7}$ meter/second, hence $\beta=0.0667$ and $\gamma=1.0022$.

In this basic consideration electron gun with plasma cathode, from which 9 A were extracted at $1 \mathrm{KeV}$ through a $6 \mathrm{~mm}$ aperture ${ }^{5}$, is considered. Based on these parameters the electron density $\mathrm{n}$ can be computed from $n=I / \mathrm{Aev}$, where $\mathrm{I}$ is electron beam current, e elementary charge and $\mathrm{A}$ is extraction aperture area. Immediately after extraction, the electron density is about $n \approx 10^{11} \mathrm{~cm}^{-3}$. Balk electrons energy spread before extraction was about $0.1 \mathrm{eV}$. Due to kinematic compression ${ }^{6}$, energy spread of the accelerated electrons $\mathrm{T}_{\mathrm{e}}$ is $T_{e}=T * 0.5(\mathrm{~T} / \mathrm{U})^{1 / 2}$, where $\mathrm{T}$ is thermal spread of unaccelerated electrons. For $\mathrm{T}=0.1 \mathrm{eV}$ and $\mathrm{U}=1 \mathrm{KeV}, \mathrm{T}_{\mathrm{e}}=5 \times 10^{-4} \mathrm{eV}$.

Calculations of single-pass electron beam cooling of the ion beam, at the end of the EBIS-RFQ-LINAC system, are repeated in this note. The computations are based on the plasma physics test particle model ${ }^{7-9}$. In addition to indication of feasibility, it is shown that there is reasonably good agreement between electron beam cooling results based on 
the plasma physics test particle model and electron beam cooling calculations using Parkhomchuk's empirical formula ${ }^{10}$.

Schematic of a possible configuration is shown in figure 1, which is similar to figure 5 of



Figure 1 sketch of a cooling setup involving electron, proton, and gold ion beams.

reference 1, except for the addition of a space-charge neutralizing proton beam. In this embodiment, the (heavy) gold ion beam and electron beams are concentric, while the proton beam is to be merged into the electron and gold ion beams. Cooling of the gold ions by the electron beam is to take place in the solenoid. The proton beam is functioning as a space charge neutralizer. Usually beam-merging optics is non-trivial at best, however, in this particular case, the proton beam need not be of high quality, since it will have no affect on the gold beam during a single pass through the solenoid (as it is shown in section IId). And the gold ion beam must be handled properly after cooling, not-theleast during separation from the electron and proton beams.

\section{Magnetized Cooling}

Cooling time (and ion heating) values computed in the previous notes ${ }^{1,2}$ are repeated, slightly modified (to account for a newer data) for this case, in which ion transverse velocity spread is dominant. Parkhomchuk's empirical formula ${ }^{10}$ and formulas ${ }^{11,12}$ based on plasma physics test particle model ${ }^{7-9}$ are used to compute cooling time. Furthermore, it is shown that unlike the currently available magnetized cooling theories ${ }^{13}$, which do not always agree with experiments ${ }^{14}$, the theoretically derived formula based on plasma physics test particle model is in excellent agreement with Parkhomchuk's empirical formula. Furthermore, the test particle model has had experimental verification ${ }^{15-17}$.

\section{IIa Test Particle Model}

Starting from the Liouville equation, Rostoker and Rosenbluth ${ }^{7}$ obtained a chain of equations by integrating out the coordinates of all but one, two, etc., particles. One "test" particle was singled out initially. All other "field" particles were assumed to be initially in thermal equilibrium. In the absence of external fields, the chain of equations was 
solved by expanding in terms of the "plasma parameter", which is basically the number of particles in a Debye sphere. For the time evolution of the distribution function of the test particle, an equation whose asymptotic form is of the usual Fokker-Planck type was obtained. It is characterized by a frictional-drag force that decelerates the particle, and a tensor that produced acceleration/deceleration and diffusion in velocity space.

Basically, the Rostoker and Rosenbluth paper $^{7}$ considered an ensemble of plasmas. The density in phase space satisfied the Liouville equation. By integrating out the coordinates of all particles but one, but two, etc., they obtained a chain of equations for one-body, two-body, etc., functions. Kadomtsev ${ }^{18}$ had previously discussed plasma kinetics in terms of chains in considering the general problem of transport theory. In the test particle model there is some loss in generality, which is compensated by a substantial gain in tractability.

Norman Rostoker continued to refine the test particle model in subsequent papers ${ }^{8,9}$ to a point where exact equations were written ${ }^{11}$ by Trubnikov for a Maxwellian field particle distribution.

Pertinent (to this case) relaxation rates $v^{\mathrm{i} / \mathrm{e}}$ in $\sec ^{-1}$ (ion test particle in a background of field electrons) are given in the following equations

$$
\begin{aligned}
& \frac{d}{d t} \overrightarrow{v_{i}}=-v_{s}^{i / e} \overrightarrow{v_{i}} \\
& \frac{d}{d t}\left(\overrightarrow{v_{i}}-\overline{v_{i}}\right)_{\perp}^{2}=v_{\perp}^{i / e} \overrightarrow{v_{i}^{2}} \\
& \frac{d}{d t}\left(\overrightarrow{v_{i}}-\overline{v_{i}}\right)_{\|}^{2}=v_{\|}^{i / e} \overrightarrow{v_{i}^{2}}
\end{aligned}
$$

Velocities are denoted by $v$ while rates are indicated by $v$. Subscripts $(s, \perp, \& \|)$ denote slowing down, transverse diffusion in velocity space and parallel diffusion in velocity space respectively. Averages are performed over an ensemble of test particle distributions for a Maxwellian field particle distribution. Exact formulas exist for relaxation rates ${ }^{11,12}$, which can be written as,

$$
\begin{aligned}
& v_{s}^{i / e}=\left(1+\frac{M}{m}\right) \psi(x) v_{0}^{i / e} \\
& v_{\perp}^{i / e}=2\left[\left(1-\frac{1}{2} x\right) \psi(x)+\psi^{\prime}(x)\right] v_{0}^{i / e} \\
& v_{\|}^{i / e}=[\psi(x) / x] v_{0}^{i / e}
\end{aligned}
$$

where $v_{0}^{i / e}=4 \pi Z^{2} e^{4} \lambda n / M^{2} v_{i}^{3}$; $\mathrm{x}$ is essentially the ratio of the test particle (ion) energy to the field particle (electron) temperature. $Z$ is ion charge state, e elementary charge and $\lambda$ is the Coulomb logarithm.

$$
\psi(x)=\frac{2}{\sqrt{\pi}} \int_{0}^{x} t^{1 / 2} e^{-t} d t \text { and } \psi^{\prime}(x)=\frac{d \psi}{d x}, \text { (here e is not the elementary charge) }
$$


In cases where $\mathrm{x}>>1$ or $\mathrm{x}<<1$, (i.e. for very fast or very slow test particles) simpler limiting forms of the relaxation rates exit ${ }^{12}$. These equations are utilized in the next subsections for ion cooling and ion heating computations.

\section{IIb Magnetized Cooling Calculations}

In case of a magnetic field, which is sufficiently strong such that at large impact parameters electron-ion collisions are adiabatic with respect to electron Larmor rotation, the electron transverse degree of freedom no longer factors in the kinetics of collisions. Under such conditions, electron beam cooling efficiency is determined by the longitudinal electron thermal spread, which is orders of magnitude smaller than the transverse spread.

Commonly "accepted" theoretical magnetized friction forces, for computing cooling times, in the sub field of electron beam cooling rates were derived by Derbenev and A.N. Skrinsky ${ }^{13}$.

$$
F_{\|}=-\frac{2 \pi e^{4} Z^{2} n \lambda}{M} \cdot \frac{3 v_{i \perp}^{2}}{v_{i}^{5}} v_{i \|}
$$

and

$$
F_{\perp}=\frac{2 \pi e^{4} Z^{2} n \lambda}{M} \bullet \frac{2 v_{i \perp}^{2}-2 v_{i \|}^{2}}{v_{i}^{5}} v_{i \perp}
$$

where subscripts $\|$ and $\perp$ refer to friction forces and to velocity spreads in the parallel and normal directions. $\mathrm{Z}$ is ion charge state, $\mathrm{n}$ is electron density, and $\lambda$ is the Coulomb logarithm.

Cooling rate, which is proportional to ion energy $\mathrm{E}$ loss rate to electrons, is defined as ${ }^{10}$ $\frac{d E}{d t}=F \bullet V$. Hence, $\tau=\frac{M v_{i}}{F}$

Substituting for $\mathrm{F}$ in equation 6 from equations 4 and 5, expressions for cooling times are obtained.

Nevertheless, Parkhomchuk ${ }^{10}$ claims that an empirical formula is in much better agreement with computer simulations of fully magnetized cooling for magnetic fields of up to $4 \mathrm{kG}$. Additionally, experiments ${ }^{19}$ and computer simulations ${ }^{20}$ showed reasonable agreement with Parkhomchuk ${ }^{10}$. Even though in this case magnetic fields are much larger and the assumption of absolute magnetization is most likely valid, Parkhomchuk's empirical formula should be used ${ }^{14}$. In this case, increase in electron beam thermal spread due to space charge ${ }^{21}$ is still lower than ion thermal spread ${ }^{14}$ (a simple estimate of the electron beam thermal spread due to the electrostatic space charge potential ${ }^{21} e^{2} n^{1 / 3}$ in cgs units yields an energy spread of $6.68 \times 10^{-4} \mathrm{eV}$, which is orders of magnitude lower than the ion beam thermal spread).

Magnetized friction force and cooling time based on the empirical formula ${ }^{10}$, in cgs units, are (for the case of hot ions and cold electron) 
$F=v_{i} \frac{4 Z^{2} e^{4} n \lambda}{m v_{t h i}^{3}}$

and cooling time

$\tau_{c}=\frac{M v_{i}}{F}=\frac{M v_{i} m v_{t h i}^{3}}{4 Z^{2} e^{4} n \lambda v_{i}}$

where $v_{i}$ and $v_{\text {thi }}$ are ion beam velocity and ion thermal spread respectively. The appropriate Coulomb logarithm in this case is

$$
\lambda=\ln \left(\frac{\left[\frac{v_{t h i}}{\omega_{p e}}\right]}{\rho_{e}}\right)
$$

where $\omega_{\mathrm{pe}}$ and $\rho_{\mathrm{e}}$ are electron plasma frequency and gyro-radius respectively. In this case the perpendicular ion thermal spread is used in equations 8 and 9, since it is larger by a factor of 5. For parameters given in the introduction section

$\lambda=3.4$ and $\tau=6 \times 10^{-8} \mathrm{sec}$

Based on this cooling time computed from equations 8 and $9\left(2 \times 10^{7}\right.$ meter/second $\mathrm{x}$ $\left.6 \times 10^{-8} \mathrm{sec}\right)$, a cooling length of 1.2 meter is needed.

Next the magnetic field needed to ensure full magnetization is estimated. If the magnetic field is not high enough, adiabaticity condition for low impact parameters collisions can be violated, in which case friction forces must be computed (and added up) for two ranges of impact parameters. Scaling up from previously analyzed cases ${ }^{21}$, maximum cooling decrement can be reached for magnetic fields of/or exceeding $2.4 \mathrm{~T}$, which is the magnetic field of choice.

Since the ion beam density (of $\mathrm{n}_{\mathrm{i}}=8 \times 10^{7} \mathrm{~cm}^{-3}$ ) is orders of magnitude lower than the electron density of $n \approx 10^{11} \mathrm{~cm}^{-3}$, cooling the ions will have negligible effect on the equilibrium electron temperature. Therefore under unmagnetized full thermal equilibrium, parallel ion temperature will be reduced to the electron temperature. But in a magnetized case the process is more complex due to freedom of motion restrictions imposed by the magnetic field.

Transverse thermal equilibrium $T_{i \perp}$ is given $b^{21}$

$$
T_{i \perp}=5 Z e^{2} n^{1 / 3}\left(\sqrt{4 \pi} \frac{\tau_{0} \Omega_{e}^{2}}{\omega_{p e}}\right)^{1 / 3}
$$

in cgs units, where $\tau_{0}$ is time an ion spends in the electron beam, $\omega_{\mathrm{pe}}$ and $\Omega_{\mathrm{e}}$ are electron plasma and cyclotron frequencies respectively. For a magnetic field of 2.4 Tesla, and $\tau_{0}$ of $6 \times 10^{-8} \mathrm{sec}$, the perpendicular ion total velocity spread is reduced to $7.9 \times 10^{3} \mathrm{~m} / \mathrm{s}$, and the transverse momentum (full) spread to 


$$
\Delta p_{\perp} / p \approx 3.9 \times 10^{-4}
$$

Basically, the transverse momentum spread, and hence ion beam emittance, can be reduced by about an orders of magnitude. And, the parallel momentum spread is reduced by an order of magnitude.

Finally, cooling calculations are made with test particle model plasma relaxation formalism. And subsequently, it is shown that Parkhomchuk's empirical formula is in excellent agreement with the test particle model (ion slowing down) relaxation time.

Since the ion beam density ( of $n_{i}=8 \times 10^{7} \mathrm{~cm}^{-3}$ ) is low, intra beam scattering (IBS) is insignificant during this cooling process. But, the electron density (of $n \approx 10^{11} \mathrm{~cm}^{-3}$ ) is many orders of magnitude higher than the ion density. Therefore, interactions with electrons dominate velocity space diffusion (relaxation). In this case the use of plasma physics formulas is fully justified to compute ion velocity space diffusion for the following reasons.

Given ion and electron beam parameters, the Debye length $\lambda_{D}=7.43 \times 10^{-4} \mathrm{~cm}$, hence there are about 1346 Debye lengths in a beam diameter. And, there are about 182 electrons in a Debye sphere. Electron gyro-radius is $3.13 \times 10^{-5} \mathrm{~cm}$. Hence, there are almost 32,000 electron gyro-radii in a beam diameter. Electron gyro-frequency is $6.72 \times 10^{10} \mathrm{~Hz}$ in this magnetic field of 2.4 Tesla. During an interaction time (computed above) of $\tau=$ $6 \times 10^{-8} \mathrm{sec}$, an electron completes $\mathbf{4 0 3 2}$ gyrations. No past, existing, or future (planned) electron beam cooler has parameters where beam diameter to gyro-radius and Debye length ratios, as well as the number of electron gyrations are such large numbers. Therefore, classical plasma physics formalism is fully justified in this analysis. In this magnetic field ion gyro-frequency is about $6 \mathrm{MHz}$, i.e. ion gyration period is over a factor of 5 larger than the longest interaction (cooling) time. So ions are not magnetized.

Ion inter-particle distance is $2.3 \times 10^{-3} \mathrm{~cm}$, i.e. larger than 3 Debye lengths. Therefore, ions are totally shielded from each other. Hence, their interaction is solely with electrons. Therefore, the test particle model, in which relaxation rates of test particles steaming through a background of field particles are computed, is particularly suitable in this application. Furthermore, relaxation rates for a single ion (test particle), whose energy equals ion beam thermal spread, streaming through cooling electrons (field particles), are a reasonable approximation of the ion beam velocity space relaxation rates. Norman Rostoker $^{7-9}$ originated the test particle model, which for a Maxwellian of field particle distribution, exact formulas ${ }^{11}$ were derived; and, expressions exist ${ }^{12}$ for cases where test particle energy is much smaller or much larger than field particle energy. Magnetic field effects $^{7,22}$ are accounted for through the Coulomb logarithm. Furthermore, the test particle model has been verified ${ }^{15-17}$ experimentally.

For simplicity, computations are performed in the beam rest frame, since $\gamma=1.0022$, corrections to time dilations are minuscule. Pertinent slowing done relaxation rate $v_{s}^{i / e}$ in $\mathrm{sec}^{-1}$ (ion test particle slowing down in a background of field electrons) is given in the following equation 
$v_{s}^{i / e}=1.7 \times 10^{-4} \mu^{1 / 2} n Z^{2} \lambda \varepsilon^{-3 / 2}$


spread in the perpendicular direction is much larger) i.e. it is the energy of "test" representative ion. Hence, cooling time

$\tau_{c}=\tau_{s} \approx 1 / v_{s}^{i / e} \approx 5.35 \times 10^{-8} \mathrm{sec}$,

which implies a cooling length of about 1 meter! The difference between this value and that obtained from Parkhomchuk's empirical formula is about $11 \%$.

Substituting numerical values for the constants $\mathrm{m}$ and $\mathrm{e}$, cooling time computed from Parkhomchuk's empirical formula, equation 8 can be written as

$\tau_{c}=4.28 \times 10^{9} \frac{M v_{t h i}^{3}}{Z^{2} n \lambda}$

Again thermal spread is used to be consistent with the test particle model.

Based on equations 11 and 12 , cooling time $\tau_{\mathrm{c}}$ can be written as $\tau_{c}=5.88 \times 10^{3} \frac{\varepsilon^{3 / 2}}{Z^{2} n \lambda \mu^{1 / 2}}$ in mixed (fusion plasma; mostly cgs and eV) units. But $\mu \equiv \frac{M}{m_{p}}$

where $\mathrm{m}_{\mathrm{p}}$ is the proton mass and $\varepsilon \equiv \frac{1}{2} M v_{t h i}^{2}$. Hence, equation 12 can be written as $\tau_{c}=5.88 \times 10^{3} \frac{\left(\frac{1}{2}\right)^{3 / 2} m_{p}^{1 / 2} M^{3 / 2} v_{\text {thi }}^{3}}{Z^{2} n \lambda M^{1 / 2}}(\sqrt{2})^{3}\left(1.6 \times 10^{-12} \frac{\mathrm{erg}}{\mathrm{eV}}\right)^{-3 / 2}$

or $\tau_{c}=3.76 \times 10^{9} \frac{M v_{t h i}^{2}}{Z^{2} n \lambda}$

Comparison between equation 14 and equation 13 yields very good agreement between cooling time computed from Parkhomchuk's empirical formula and cooling time computed from plasma relaxation based on the test particle model.

\section{IIc Ion Heating by Beam Electrons}

As it was mentioned before, this is an ideal case for applying the test particle model. For simplicity, the next computations are performed in the beam rest frame, since $\gamma=1.0022$, corrections to time dilations are minuscule.

Comparing equations 2 and 3 to equation 1, it is obvious that on a long time scale ion cooling dominates, since ion velocity slows-down with time $t$, while ion heating develops as $\sqrt{t}$, as is the case in multi-pass cooling ${ }^{14}$. In those cases, this is supposed to be the reason, why electron beam cooling works under existing cooling theories ${ }^{14}$. 
Pertinent relaxation rates $v^{\mathrm{i} / \mathrm{e}}$ in $\sec ^{-1}$ (ion test particle in a background of field electrons; ions faster than electrons), based the test particle model, are defined in the following equations $^{12}$

$v_{\perp}^{i / e}=1.8 \times 10^{-7} \mu^{-1 / 2} n Z^{2} \lambda \varepsilon^{-3 / 2}$

$v_{\|}^{i / e}=1.7 \times 10^{-4} \mu^{1 / 2} n Z^{2} \lambda T_{e} \varepsilon^{-5 / 2}$

Units are cgs and eV. $\mathrm{T}_{\mathrm{e}}$ is the electron, $\mu$ ion to proton mass ratio. Since electron are magnetized, the Coulomb logarithm ${ }^{7,22}$ (b is the smallest impact parameter) is

$\lambda=\ln \left(\frac{\rho_{e}}{b}\right) \approx 3.2$

Comparing denote slowing down rate (equation 11) to transverse diffusion in velocity space and parallel diffusion in velocity space rates (equations 15 and 16 respectively), it becomes clear that cooling rate is orders of magnitude faster. In the case of transverse velocity space diffusion,

$\frac{v_{s}^{i / e}}{v_{\perp}^{i / e}}=\frac{1.7 \times 10^{-4} \mu^{1 / 2} n Z^{2} \lambda \varepsilon^{-3 / 2}}{1.8 \times 10^{-7} \mu^{-1 / 2} n Z^{2} \lambda \varepsilon^{-3 / 2}} \cong 1.7 \times 10^{5}$

From equations 18, and the cooling (slowing down) time computed earlier, $\tau_{\perp} \approx 1.7 \times 10^{5} \bullet 6 \times 10^{-8} \approx 1 \times 10^{-2} \mathrm{sec}$, i.e., about $10 \mathrm{msec}$.

$\frac{v_{s}^{i / e}}{v_{\perp}^{i / e}}=\frac{1.7 \times 10^{-4} \mu^{1 / 2} n Z^{2} \lambda \varepsilon^{-3 / 2}}{1.7 \times 10^{-4} \mu^{1 / 2} n Z^{2} \lambda T_{e} \varepsilon^{-5 / 2}}=\frac{\varepsilon}{T_{e}} \approx 1.87 \times 10^{5}$

Equation 19 represents again a very large number. Hence, $\tau_{\|} \approx 2 \times 10^{5} \bullet 6 \times 10^{-8} \approx 1.1 \times 10^{-2} \mathrm{sec}$, i.e. about $11 \mathrm{msec}$.

Thus, velocity space diffusion relaxation times that are orders of magnitude longer than the computed cooling times. Equations 16 and 19 represent a major departure from currently used theories.

\section{IId Space Charge Heating; Proton Beam Neutralization}

The intense, low energy, electron beam, which is being considered, has a large space charge, whose resultant electric field affects mobility ion. And, spatial variations in electric field (zero or very small in electron beam center, while large at outer radii) affect ions differently at different radii. These two effects can lead to modification (perturbation) of both velocity and configuration space ion distributions, i.e., heating. Mathematically, this process could probably be treated by including collisions in the Boltzmann equation, using the Fokker-Planck method and solving for diffusion in both velocity and configuration space; a rather difficult task at best. Additionally, this space charge also heats the electron beam and reduces its cooling effectiveness.

Mitigating this problem has a simple, straight forward, solution: co-propagate a $2 \mathrm{MeV}$ proton beam with the electron beam, which will neutralize the space charge and eliminate all associated problems. Since the proton beam is also co-propagating with the gold ion beam, no charge exchange (gold ions losing electrons) can occur (no resonant charge exchange between co-moving unlike particles). In addition, any velocity space relaxation 
processes between the protons and the gold ions are too slow compared to electron processes. Slowing down and parallel diffusion due to protons gold ions interactions are four orders of magnitude slower than due to interactions with electrons ${ }^{11,12}$, while perpendicular diffusion is slow in both cases. Also, since all three beams propagate with the same velocity no instabilities are expected, since there is no free energy source. Basically, co-injecting a proton beam will solve the space charge problem; more in the discussion section.

\section{IIe Coulomb Logarithm}

In classical plasma physics the Coulomb logarithm is defined as the ration of largest to smallest deflection angle (can be found in many basic plasma books like reference 21 for example). The largest possible deflection $\left(180^{\circ}\right.$ scattering $)$ is produced by the smallest impact parameter, which is basically the ratio of electrical potential energy to particle kinetic energy, i.e. how close can two particles be. The largest impact parameter is of course the Debye shielding length. It all works very for infinite homogenous unmagnetized plasmas with isotropic temperature. But as it can be seen from section II, deviation from these conditions can lead to unrealistic results.

To be consistent with classical Boltzmann theory, the largest impact parameter is taken to $\mathrm{be}^{7,22}$ the electron gyro-radius. Parkhomchuk's empirical formula ${ }^{10}$ also sets the electron gyro-radius as the largest impact parameter. Nevertheless, the electron gyro-radius should not be set as the largest impact parameter in a cavalier way in any magnetized plasma. In cold, high-density plasmas in low to moderate magnetic fields, Debye lengths can be shorter than electron gyro-radii. Hence the Debye length must be set as the largest impact parameter. In this case the Debye length is $\lambda_{\mathrm{D}}=7.43 \times 10^{-4} \mathrm{~cm}$, which is much longer than the electron gyro-radius of $3.13 \times 10^{-5} \mathrm{~cm}$. Therefore, setting the electron gyro-radius as the largest impact parameter is fully justified in this analyzed case.

\section{Pertinent Physics Issues}

A number of pertinent physics issues are evaluated next. First topic is maintaining electron beam parameters. Second subject matter is adverse effects the electron beam (or electron gun) might have on the gold ions. Third topic is whether electron gun generated gas is tolerable.

\section{IIIa Magnetic Field Required for Electron Beam Equilibrium and Stability}

As it is shown in the next section, current density of the extracted electron beam exceeds the (structural) Child Langmuir law limit. Hence, rapid expansion of the electron beam occurs, unless the expansion is prevented by a magnetic field. For a square electron density profile, the electric field $\mathrm{E}_{\mathrm{e}}$ at the outer beam radius $\mathrm{R}$ is given in mks units by

$$
E_{e}=-I / 2 \pi \varepsilon_{0} v R
$$


Neglecting kinetic pressure (will be become obvious a posteriori that the assumption is correct), to contain the electron beam, magnetic pressure must balance pressure generated by the electric field. Hence the needed magnetic field B can be found from ${ }^{23}$

$0.5 \varepsilon_{0} E_{e}^{2}=\frac{B^{2}}{2 \mu_{0}} \quad$ or $\quad B^{2}=E_{e}^{2} / c^{2}$

From equation 20, the electric field $\mathrm{E}_{\mathrm{e}}=2.7 \times 10^{6} \mathrm{~V} / \mathrm{m}$. Therefore from equation $21 \mathrm{a}$ magnetic field of about 90 Gauss is sufficient to contain the electron beam. Since the electron beam is space charge dominated, thermal spread contributions to its pressure are negligible. A more stringent magnetic field requirement is imposed by plasma stability, which necessitates a magnetic field that satisfies ${ }^{24}$

$\frac{\omega_{p e}^{2}}{\Omega_{e}^{2}} \leq 1$

Equation 22 yields a minimal magnetic field requirement of about $2 \times 10^{3}$ Gauss or 0.2 Tesla. These magnetic fields are small compared to the 2.4 Tesla magnetic field, which maximizes the cooling decrement. Hence electron beam confinement and stability is not an issue.

At a magnetic field of 2.4 Tesla, electron gyro-frequency is $6.72 \times 10^{10} \mathrm{~Hz}$. Therefore, electrons complete over 4032 gyrations in $60 \mathrm{nsec}$. Hence, beam electrons are magnetized during the ion cooling process.

\section{IIIb Ion Loss Due to Recombination}

Due to interactions with beam electrons ion inelastic interactions are: electron capture and ionization. But due to the relative low energy differential, the only ion loss mechanism is due to recombination, which was studied extensively ${ }^{25-27}$ for electron coolers. Rate coefficient for recombination $\alpha$ is ${ }^{23}$

$$
\alpha=3.02 \times 10^{-13} \frac{Z^{2}}{\sqrt{T_{e \perp}}}\left[\ln \left(\frac{11.32 Z}{\sqrt{T_{e \perp}}}\right)+0.14\left(\frac{T_{e \perp}}{Z^{2}}\right)^{1 / 3}\right]
$$

from which reaction rate can be computed and ion lifetime $\tau_{\text {rec }}$ is ${ }^{26,27}$

$$
\tau_{\text {rec }}=\frac{\gamma}{n \alpha}
$$

Equations 23 and 24 are in cgs units except for $\mathrm{T}$, which is in $\mathrm{eV}$. The assumption in deriving equation 23 that $T_{e \perp}>>T_{e \|}$ is valid for our case too (all is done is laboratory frame). For our parameters $\boldsymbol{\tau}_{\text {rec }}$ is about 1.5 msec, which is orders of magnitude longer than any computed cooling time. Additionally, electron capture is suppressed in such a large magnetic field. Hence, electron recombination is not an issue in this process.

Other channels of recombination like three body collisional recombination or dielectronic recombination have extremely low probability ${ }^{28}$. Cross $\operatorname{section}^{29,30}$ for the latter, for example, is usually of the order of $10^{-19} \mathrm{~cm}^{2}$ or less. The low cross sections combined with 10 's nsec interaction time and a mean free path of $10^{8} \mathrm{~cm}$ render these processes unimportant. 


\section{IIIc Charge Exchange}

One of the electron guns that is being considered is hollow cathode plasma cathode ${ }^{5}$ (to be described in the next section; relevance of this paragraph will become apparent in the next section). Pressure in the electron gun ${ }^{3}$ is about $10^{-5}$ Torr of argon gas. High charge exchange cross sections are usually of the order of $10^{-14} \mathrm{~cm}^{2}$, for which the mean free path is $336 \mathrm{~cm}$. However, inside the hollow cathode the pressure is about $10^{-2}$ Torr. In this pressure the mean free path is less than $0.3 \mathrm{~cm}$. Therefore, ion beam injection through the hollow cathode in current embodiment is not an option. In the electron gun extractor background gas pressure is under $10^{-5}$ Torr; hence the mean free path is a longer than $400 \mathrm{~cm}$. Outside the extractor pressure is $10^{-7}$ Torr, where charge exchange is no longer an issue. Nevertheless, switching the hollow cathode discharges to hydrogen operation can eliminate charge exchange as an issue, since hollow cathode discharges generate plasma that is $100 \%$ ionized, and since protons have no electrons "to give." Electron capture by protons from highly ionized gold ions is negligible.

A more suitable electron gun could be an electron gun with carbon fiber cathode ${ }^{31}$, which have generated close to $1 \mathrm{MA}$ of electron current. More recently ${ }^{32}$ currents of up to $2 \mathrm{kA}$ at $2 \mathrm{kV}$ were obtained in microseconds long pulses. Depending on the current generated, pressure during the electron beam pulse can be between $10^{-3}$ to $10^{-6}$ Torr (or even lower where large pumping capability is available). Since the needed electron beam currents are well below $100 \mathrm{~A}$, pressures below $10^{-6}$ Torr, where charge exchange is not an issue, are expected.

\section{IIId Other Plasma Instabilities}

Based on equation 22, the electron beam should be stable for an axial magnetic field of 2.4 Tesla. If the electron beam is stable, there should, in principle, be no other instabilities. The only possible plasma instability might be to the ions (like a rotating two stream instability). Like all beam instabilities, it has a density threshold. Since the ion density is more than three orders of magnitude lower than the electron density, there should be no beam instabilities.

\section{Some Comments Electron Beam Issues}

Electron beam technology to be discussed in a subsequent note; in this subsection further explanation is given for electron beam parameters used previously. Electron beam parameters used in the above computations are based on a novel electron gun with a plasma cathode was developed at BNL ${ }^{5,33,34,35}$, during the late 1980's till the mid 1990's. Parallel thermal spread is based on fairly accurate, repeatable measurements that were repeated over a few years. Perpendicular thermal spread was estimated from beam envelope measurements ${ }^{35}$ to be less than $0.5 \mathrm{eV}$. The poorer resolution is due to the dominance of space charge effects in the beam expansion. Nevertheless, due to 
suppression of electron transverse degree of freedom in case of a magnetic field, which is sufficiently strong such that at large impact parameters electron-ion collisions are adiabatic with respect to electron Larmor rotation, transverse electron energy plays only a minor role in electron beam cooling. Quantitatively as it can be seen from equations 9 and 17, transverse thermal spread enters only in the Coulomb logarithm as transverse velocity. Effect of any increase in transverse thermal energy spread on electron beam cooling is minimized, since square root following by the log of the increase is taken. For example, if the transverse thermal energy spread is an order larger, the numerical effect on the Coulomb logarithm (and hence on cooling length) is about $20 \%$.

Kinematic compression formula ${ }^{6}$ employed in the introduction, is commonly used in spectroscopy of moving excited ion beams. However, kinematic compression does depend on system particulars. In reference 19, there is a thorough discussion of kinematic compression in electron guns. By averaging over the distribution of accelerated electron, an expression is obtained for energy spread of the accelerated electrons $T_{e}$ to be, $T_{e}=\frac{T^{2}}{2 U}$, where $\mathrm{T}$ is thermal spread of unaccelerated electrons. For $\mathrm{T}=0.1 \mathrm{eV}$ and $\mathrm{U}=1$ $\mathrm{KeV}, \mathrm{T}_{\mathrm{e}}=5 \times 10^{-6} \mathrm{eV}$. However, due to electron beam space charge, there is a factor of about $\mathrm{Ce}^{2} \mathrm{n}^{1 / 3}$ increase in longitudinal thermal spread; $\mathrm{C}$ is a factor of order unity that depends on the magnetic field (in our particular case, $\mathrm{C}$ is most likely less than 1).

$T_{e} \approx \frac{T^{2}}{2 U}+C e^{2} n^{1 / 3}$

For this electron beam parameters, space charge is the dominant factor and (for $\mathrm{C}=1$ ), $T_{e} \approx 6.68 \times 10^{-4} \mathrm{eV}$, basically the same value obtained from the formula used in spectroscopy.

\section{Discussion}

Other than Parkhomchuk's empirical formula, which is used to calculate cooling rates, most computations in this note are based on the test particle model plasma physics formalism. Given ion and electron beam parameters, the Debye length $\lambda_{D}=7.43 \times 10^{-4} \mathrm{~cm}$, hence there are about 1346 Debye lengths in a beam diameter. And, there are about 182 electrons in a Debye sphere. Electron gyro-radius is $3.13 \times 10^{-5} \mathrm{~cm}$. Hence; there are almost 32,000 electron gyro-radii in a beam diameter. Electron gyro-frequency is $6.72 \times 10^{10} \mathrm{~Hz}$ in this magnetic field of 2.4 Tesla. During an interaction time (computed above) of $\tau=6 \times 10^{-8} \mathrm{sec}$, an electron completes $\mathbf{4 0 3 2}$ gyrations. In this magnetic field ion gyro-frequency is about $6 \mathrm{MHz}$, i.e. ion gyration period is over a factor of 5 larger than the longest interaction (cooling) time. So ions are not magnetized. Therefore, plasma physics formalism, especially when based on the test particle model, is fully justified in this analysis. Answer to the question posed in the title, on whether single pass cooling is possible, is affirmative.

Final theoretical questions to be answered in this note are: what is the reason for difference in theoretical electron beam cooling results and which results are correct (or 
which theory is more realistic)? Existing electron beam cooling theories are based on multiple small angle binary Coulomb collisions. Most seminal papers ${ }^{10,13,20}$ on this subject start with a friction force formula referenced to the original proposal for electron beam cooling ${ }^{36}$ by Budker. However, that paper just quotes results from an earlier reference ${ }^{37}$ by Belyaev and G.I. Budker, where energy transfer time, due to friction force for multiple small angle binary Coulomb collisions, is derived. Based on that formalism, Budker $^{36}$ proceeds to evaluate electron beam cooling for protons and antiprotons.

Major difference between electron beam cooling derivations by Budker $^{36}$ as well as Derbenev and Skrinsky ${ }^{13}$ and plasma relaxation formulas is that the first is based on multiple small angle binary Coulomb collisions, while in the latter a test particle interacts simultaneously with many particles (within a Debye sphere). Common to the Boltzmann equation, which describes relaxation of gas atoms and molecules (due to short range forces), Chadrasekhar's ${ }^{38}$ multiple long-range binary collisions between stars, and Spitzer's ${ }^{39}$ multiple long-range binary Coulomb collisions, is particle discreteness. Conversely, in the test particle model, just as in the Vlasov fluid, the number of particles in a Debye sphere is very large such that $\mathrm{e} \rightarrow 0, \mathrm{n} \rightarrow 0$, but $\mathrm{e} / \mathrm{m}=\mathrm{constant}$ as well as $\mathrm{n} \bullet \mathrm{e}=$ constant. In theories based on the Vlasov equation, correlation (interactions) among particles occurs via wave-particle interactions. In the test particle model individual particle effects emerge from hierarchy of statistical equations derived from the Liouville equation to the "plasma parameter" $\left(1 / n \lambda_{D}^{3}\right)$, i.e. the number of particles in a Debye sphere. Which theoretical approach to electron beam cooling computation is more appropriate depends on the level of particle discreteness i.e. the number of particles in a Debye sphere. In this particular case, there are 182 particles $(>>1)$ in a Debye sphere. Hence, the test particle model is more realistic.

Finally here are some comments regarding co-propagation of a proton beam. During a talk given by the author on $1 / 23 / 2009$, Alexei Fedotov ${ }^{40}$ pointed out that he had just repeated the cooling calculation (computerized; using Parkhomchuk's empirical formula) and found agreement with reference 1 calculations, when only ion velocity spread is considered. Inclusion of space charge effects on electrons increases the cooling length by a factor of $2-3$; still not a show stopper. However, when space charge effects on the ions are considered, the cooling length increases to about $2-3 \mathrm{~km}$; definitely a show stopper! Subsequently, a solution was found: co-injection of $2 \mathrm{MeV}$ protons. There are two feasible possibilities: injecting the protons from a proton diode similar to those generated at $\mathrm{NRL}^{41}$, but with higher energy than their $1.2 \mathrm{MeV}$, and much lower current than their 1 MA (should be a relatively straight forward task). Or, a better possibility is to mount a conventional, clean proton ion source "floating" on top of a positively charge Marx generator to obtain the needed proton beam. Co-injection of equal $2 \mathrm{MeV}$ proton current will solve the space-charge problem associated with the electron beam.

Interesting physics regardless of the particular application!

\section{References}


1. Ady Hershcovitch, "Emittance Reduction between EBIS LINAC and Booster by Electron Beam Cooling; Is Single Pass Cooling Possible?" C-A/AP note \#303 April 2008 (unpublished).

2. Ady Hershcovitch, "Part II/Addendum Electron Beam Cooling between EBIS LINAC and Booster; Is Single Pass Cooling Possible?" C-A/AP note \#317 July 2008 (unpublished).

3. Ady Hershcovitch, "Issues Concerning High Current Low Energy Electron Beams Required for Ion Cooling between EBIS LINAC and Booster" C-A/AP note \#317 July 2008 (unpublished).

4. Deepak Raparia, private communication (2008).

5. Ady Hershcovitch, APL 68, 464 (1996).

6. S.L. Kaufman, Opt. Comm. 17, 309 (1976).

7. N. Rostoker and M.N. Rosenbluth, Physics of Fluids 3, 1 (1960).

8. N. Rostoker, Nuclear Fusion 1, 101 (1961).

9. N. Rostoker, Physics of Fluids 7, 491 (1964).

10. V.V. Parkhomchuk, NIM A 441, 9 (2000).

11. B.A. Trubnikov, "Particle Interactions in a Fully Ionized Plasma," Rev. of Plasma Phys., 1 (Consultants Bureau, N.Y.) pp. 105-204 (1965).

12. NRL Plasma Formulary, J. D. Huba, NRL/PU/6790-06-491, revised 2006.

13. Ya.S. Derbenev and A.N. Skrinsky, Plasma Physics 4, 492 (1978).

14. Alexei Fedotov private communications (2007 \& 2008).

15. J. Bowles, R. McWilliams, and N. Rynn, Phys. Rev. Letters 68, 1144 (1992).

16. J. Bowles, R. McWilliams, and N. Rynn, Physics of Plasmas 1, 3418 (1994).

17. J.J. Curry, F. Skiff, M. Sarfaty, and T.N. Good, Phys. Rev. Letters 74, 1767 (1995).

18. B.B. Kadomstev, Soviet Phys.-JETP 6, 117 (1958).

19. A.V. Fedotov, B. Gålnander, V.N. Litvinenko, T. Lofnes, A. Sidorin, A. Smirnov and A. Zieman, Physical Review E 73, 066503 (2006).

20. A. V. Fedotov, D.L. Bruhwiler, A. Sidorin, D.T. Abell, I. Ben-Zvi, R. Busby, J.R. Cary, and V.N. Litvinenko, Physical Review ST Accel. Beams 9, 074401 (2006).

21. N.S. Dikansky, V.I. Kudelainen, V.A. Lebedev, I.N. Meshkov, V.V. Parkhomchuk, A.A. Sery, A.N. Skrinsky, and B.N. Sukhina, "Ultimate Possibilities of Electron Cooling," Institute of Nuclear Physics, Novosibirsk, USSR Report, Preprint 88-61 (1988).

22. D. Montgomery, G. Joyce, and L. Turner, Physics of Fluids 17, 2201 (1974).

23. D.J. Rose and M. Clark, "Plasmas and Controlled Fusion", MIT Press, Cambridge MA (1961).

24. R.C. Davidson, "Theory of Nonneutral Plasmas", W.A. Benjamin, Inc. Advanced Book Program, Reading MA (1974).

25. M. Bell and J.S. Bell, Particle Accelerator 12, 49 (1982).

26. A. Wolf et al. NIMA 441, 183 (2000).

27. V.V. Parkhomchuk, "(Electron Cooling for RHIC)," Budker INP, Novosibirsk, Russia (2000) unpublished report.

28. H. Poth, CERN-EP/90-04.

29. P.F. Dittner, Phys. Scr. T22, 65 (1988).

30. Y. Hahn, and K.J. LaGattuta, Phys. Rep. 166, 195 (1988). 
31. R. Prohaska and A. Fisher, Rev. of Sci. Instrum. 53, 1092 (1982).

32. A. Hershcovitch, B. Johnson, F. Liu, A. Anders, I. Brown, Rev. of Sci. Instrum. 69, 798 (1998).

33. A. I. Hershcovitch, V. J. Kovarik, and K. Prelec, J. Appl. Phys. 67, 671 (1990).

34. A. Hershcovitch, J. Appl. Phys. 74, 728 (1993).

35. A. Hershcovitch, "Proceedings of the Fifth International EBIS Symposium", edited by E. D. Donets and I. P. Yudin (JINR, Dubna, EBIS-5, Russia, 1991).

36. G.I. Budker, Atomnaya Energia, 22, 346 (1967).

37. S.T. Belyaev and G.I. Budker, Doklady Akad. SSSR, 107, 807 (1956) (in Russian only).

38. S. Chadrasekhar, "Principle of Stellar Dynamics", University of Chicago Press, Chicago 1942.

39. L. Spitzer, Jr., "Physics of Fully Ionized Gases", Interscience Publishers, Inc., New York, 1956.

40. Alexei Fedotov private communication 2009.

41. Goldstein et al, PRL $\underline{40}, 1504$ (1978); Young et al, PRL 0ㅜ, 2573 (1993).

\section{Acknowledgement}

Very helpful discussions with Deepak Raparia, Ilan Ben-Zvi, Brant Johnson, Norman Rostoker and Roger McWilliams are gratefully acknowledged. Many special thanks to Alexei Fedotov for numerous stimulating and extremely helpful discussions!

Notice: This manuscript has been authored by Brookhaven Science Associates, LLC under Contract No. DE-AC02-98CH1-886 with the US Department of Energy. The Untied States Government retains, and the publisher, by accepting the article for publication, acknowledges, a world-wide license to publish or reproduce the published form of this manuscript, or others to do so, for the United States Government purposes. 